\title{
Clique Dominating Sets of Euler Totient Cayley Graphs
}

\author{
${ }^{1}$ M. Manjuri, ${ }^{2}$ B. Maheswari \\ ${ }^{1,2}$ Department of Applied Mathematics, Sri Padmavati Women's University, Tirupati, Andhra Pradesh, India.
}

\begin{abstract}
Graph Theory has been realized as one of the most flourishing branches of modern Mathematics finding widest applications in all most all branches of Sciences, Social Sciences, Engineering, Computer Science, etc. Number Theory is one of the oldest branches of Mathematics, which inherited rich contributions from almost all greatest mathematicians, ancient and modern. Using the number theoretic function Euler totient function we have defined an Euler totient Cayley graph and in this paper we study the Clique domination parameters of Euler totient Cayley graphs.
\end{abstract}

Keywords: Cayley Graph, Clique, Complete graph, Dominating clique, Euler totient Cayley Graph

\section{Introduction}

Nathanson [1] was the pioneer in introducing the concepts of Number Theory in Graph Theory, thus paved the way for the emergence of a new class of graphs, namely, Arithmetic Graphs. Cayley Graphs are another class of graphs associated with elements of a group. If this group is associated with some Arithmetic function then the Cayley graph becomes an Arithmetic graph.

The Cayley graph associated with Euler totient function is called an Euler totient Cayley graph .

\section{Cayley graph}

Let $(X,$.$) be a group and S$, a symmetric subset of $X$ not containing the identity element $e$ of $X$. The graph $G$ whose vertex set $V=X$ and edge set $E=\{(g, g s) / s \in S\}$ is called the Cayley graph of $X$ corresponding to the set $S$ and it is denoted by $G(X, S)$.

Madhavi [2] introduced the concept of Euler Totient Cayley graphs and studied some of its properties.

\section{Euler totient Cayley Graph}

For each positive integer $n$, let $Z_{n}$ be the additive group of integers modulo $n$ and $S$ be the set of all numbers less than $n$ and relatively prime to $n$. The Euler Totient Cayley graph $G\left(Z_{n}, \varphi\right)$ is defined as the graph whose vertex set $\mathrm{V}$ is given by $Z_{n}=\{0,1,2, \ldots n-1\}$ and the edge set is given by $E=\{(x, y) / x-y \in S\}$.

Clearly as proved by Madhavi [2], the Euler Totient Cayley $\operatorname{graph} G\left(Z_{n}, \varphi\right)$ is

1. a connected, simple and undirected graph,

2. $\Phi(n)$ - regular and has $n . \Phi(n) / 2$ edges,

3. Hamiltonian,

4. Eulerian for $n \geq 3$,

5. Bipartite if $\mathrm{n}$ is even,

6. Complete graph if $n$ is a prime.

The domination parameters of Euler totient Cayley graphs are studied by Uma Maheswari [3] and we present some of the results without proofs and the proofs can be found in [4].

Theorem 2.1: If $n$ is a prime, then the domination number of $G\left(Z_{n}, \varphi\right)$ is 1 .

Theorem 2.2: If $n$ is power of a prime, then the domination number of $G\left(Z_{n}, \varphi\right)$ is 2 .

Theorem 2.3: The domination number of $G\left(Z_{n}, \varphi\right)$ is 2 , if $n=2 p$ where $p$ is an odd prime.

Theorem 2.4: Suppose $n$ is neither a prime nor $2 p$. Let $n=p_{1}^{\alpha_{1}} p_{2}^{\alpha_{2}} \ldots \ldots . p_{k}^{\alpha_{k}}$ where $p_{1,} p_{2,}, p_{3}, \ldots p_{k}$ are primes and $\alpha_{1}, \alpha_{2}, \alpha_{3}, \ldots \ldots, \alpha_{k}$ are integers $\geq 1$. Then the domination number of $G\left(Z_{n}, \varphi\right)$ is given by $\gamma\left(G\left(Z_{n}, \varphi\right)\right)=\lambda+1$, where $\lambda$ is the length of the longest stretch of consecutive integers in $V$, each of which shares a prime factor with $n$.

\section{Clique Dominating Sets of Euler Totient Cayley Graphs}

The theory of domination in Graphs introduced by Ore [5] and Berge [6] is an emerging area of research today. It founds applications in communication networks, computer science etc. The concept of clique domination in graphs is introduced by Cozzens and Kelleher [7]. They established a sufficient condition for a graph to have a dominating clique in terms of forbidden subgraphs. Further they gave an upper bound on the 
clique domination number. Also Basco[8] studied dominating cliques in $P_{5}-$ free graphs and obtained bounds for clique domination number.

A simple Graph $G$ in which each pair of distinct vertices is joined by an edge is called a complete graph and is denoted by $K_{n}$.

Let $G(V, E)$ be a graph. A subset $D$ of $V$ is said to be a dominating set of $G$ if every vertex in $V-D$ is adjacent to a vertex in $D$. The minimum cardinality of a dominating set is called the domination number of $G$ and is denoted by $\gamma(G)$.

A non-empty subset $S$ of $V$ is called a clique if the induced subgraph $\langle S\rangle$ is a complete graph.

A dominating set $D$ of a graph $G$ is called a dominating clique if the induced sub graph $\langle D\rangle$ is a complete graph. The cardinality of the smallest dominating clique is called clique domination number and is denoted by $\gamma_{c l}$.

Theorem 1: The clique domination number of $G\left(Z_{n}, \varphi\right)$ is 1 , if $n$ is a prime.

Proof: Let $n$ be a prime. Then $G\left(Z_{n}, \varphi\right)$ is a complete graph. So any single vertex in $G\left(Z_{n}, \varphi\right)$ will dominate all other vertices.

Therefore $\gamma\left(G\left(Z_{n}, \varphi\right)\right)=1$.

So, if $D_{c l}=\{0\}$, then the induced sub graph $\left\langle D_{c l}>\right.$ is a complete graph with minimum cardinality. Hence $D_{c l}$ is a minimum dominating clique of $G\left(Z_{n}, \varphi\right)$.

Therefore $\gamma_{c l}\left(G\left(Z_{n}, \varphi\right)\right)=1$.

Theorem 2: If $n$ is power of a prime, then the clique domination number of $G\left(Z_{n}, \varphi\right)$ is 2 .

Proof: Consider $G\left(Z_{n}, \varphi\right)$ for $n=p^{\alpha}$ where $p$ is a prime. Then the vertex set $\mathrm{V}$ of $G\left(Z_{n}, \varphi\right)$ is given by $V=\left\{0,1, \ldots \ldots p^{\alpha-1}\right\}$. This set $\mathrm{V}$ falls into the following disjoint subsets.

1. The set $S$ of integers relatively prime to $n$,

2. The set $M$ of multiples of $p$,

3. Singleton set $\{0\}$.

Let $D_{c l}=\{0, t / G C D(t, n)=1\}$ where $t \in S$ and $t$ is a relatively prime to $n$. Then $D_{c l}$ becomes a minimum dominating set of $G\left(Z_{n}, \varphi\right)$ as in Theorem 2.2. Further the vertices 0 and $t$ are adjacent. This gives that $<D_{c l}>$ is a complete graph. So $D_{c l}$ is a dominating clique of $G\left(Z_{n}, \varphi\right)$ of minimum cardinality. Hence it follows that $\gamma_{c l}\left(G\left(Z_{n}, \varphi\right)\right)=2$.

Theorem 3: If $n=2 p$, where $p$ an odd prime, then the clique domination number of is $G\left(Z_{n}, \varphi\right)$ does not exist.

Proof: Let us consider the Euler totient Cayley Graph $G\left(Z_{n}, \varphi\right)$ for $n=2 p$, where $p$ is an odd prime. Then the vertex set $V=\{0,1,2,3, \ldots \ldots, 2 p-1\}$ falls into the following disjoint subsets.

1. The set $S$ of odd numbers which are less than $\mathrm{n}$ and relatively prime to $n$,

2. The set of $M$ of non-zero even numbers,

3. The set $D$ of numbers 0 and $p$.

As in Theorem 2.3, we know that $D=\{0, p\}$ is a minimum dominating set of $G\left(Z_{n}, \varphi\right)$.

Now we check whether $D=\{0, p\}$ is a dominating clique or not.

The vertices in $D$ are non - adjacent because $G C D(p, n) \neq 1$. This gives that $\langle D\rangle$ is not a complete graph. Further the dominating sets of $G\left(Z_{n}, \varphi\right)$ generated by $(0, p)$ are $(1, p+1),(2, p+2),(3, p+$ 3) ... $(p-1,2 p-1)$. Since $p \notin S$, it follows that the vertices in these dominating sets are non - adjacent. Hence a dominating set of cardinality 2 is not a dominating clique in $G\left(Z_{n}, \varphi\right)$. Therefore if $D_{c l}$ is a dominating clique of $G\left(Z_{n}, \varphi\right)$ then $\left|D_{c l}\right| \geq 3$.

We claim that any set of three vertices in $G\left(Z_{n}, \varphi\right)$ does not form a dominating set.

Case 1: Let $u, v, w \in S$, where $S$ is a set of odd integers which are relatively prime to $n$ and $p$ is an odd prime. Then $G C D(u, p)>1, G C D(v, p)>1, G C D(w, p)>1$. Hence the vertex $p$ is not adjacent to $u, v, w$.

That is any set of three vertices in $\mathrm{S}$ does not form a dominating set.

Case 2: Consider any three vertices $x, y, z \in M$, where $M$ is the set of non-zero even integers. Obviously the vertex 0 is not adjacent to the vertices in $M$ as their difference is an even number which is in $M$ but not in $S$.

That is any set of three vertices in $M$ does not form a dominating set.

Case 3: Suppose we consider two vertices in $S$, say $u, v \in S$ and one vertex $w \in M$. Then any vertex $x$ in $G\left(Z_{n}, \varphi\right)$ such that $|x-w|=p$ is not adjacent to the vertices $u, v$. This is because $|x-w|=p$ implies that $x$ is an odd number and its difference with $u, v$ is even and this even number does not belong to $S$. Further $x$ and $w$ are not adjacent as $|x-w|=p$ and $p \notin S$.

Similar is the case with $u, v \in M$ and $w \in S$.

Therefore any set of three vertices in $G\left(Z_{n}, \varphi\right)$ does not form a dominating set in $G\left(Z_{n}, \varphi\right)$. 
Hence $\left|D_{c l}\right| \geq 4$.

We can get a dominating set of cardinality $4 \operatorname{in} G\left(Z_{n}, \varphi\right)$. But for all choices of these vertices in $\mathrm{S}$ and $\mathrm{M}$, we can not get a complete graph on these vertices because of the definition of the sets $\mathrm{S}$ and $\mathrm{M}$.

Similar is the case with any dominating set of cardinality more than 4 .

Hence Clique domination number does not exist for the graph $G\left(Z_{n}, \varphi\right)$.

Theorem 4: Let $n=p_{1}^{\alpha_{1}}, p_{2}^{\alpha_{2}}, \ldots \ldots . p_{k}^{\alpha_{k}}$ where $p_{1}, p_{2}, p_{3,} \ldots p_{k}$, are primes and $\alpha_{1}, \alpha_{2}, \alpha_{3}, \ldots \ldots, \alpha_{k}$ are integers $\geq 1$ and $\mathrm{n}$ is neither a prime nor $2 p$. If $n$ is an even number then the clique domination number does not exist for $G\left(Z_{n}, \varphi\right)$. Otherwise the clique domination number is 3 , if $n=p_{1}^{\alpha_{1}}, p_{2}^{\alpha_{2}}$.

Proof: Let $n=p_{1}^{\alpha_{1}} p_{2}^{\alpha_{2}} \ldots \ldots . p_{k}^{\alpha_{k}}$, where $n$ is neither a prime nor $2 p$. The vertex set $V$ of $G\left(Z_{n}, \varphi\right)$ is given by $V=\{0,1,2, \ldots ., n-1\}$. Then the set $V$ falls into disjoint subsets as follows.

1. The set $S$ of integers relatively prime to $n$,

2. The set $X=\left\{S_{i}\right\}$, where $S_{i}$ is a collection of consecutive positive integers such that for every $x$ in $S_{i}$, $\operatorname{GCD}(x, n)>1$,

3. The singleton set $\{0\}$.

Let $S_{\lambda}$ be the largest set in $X$ with cardinality $\lambda$ and $S_{\lambda}=\left\{x_{1}, x_{2}, \ldots \ldots x_{\lambda}\right\}$, where $G C D\left(x_{i}, n\right)>1$ for $i=1,2, \ldots, \lambda$. then $D=\{0,1,2 \ldots . \lambda\}$ is a dominating set of minimum cardinality by Theorem 2.4 . Now two cases arise.

Case 1: Suppose $n$ is an even number. Since the vertices in $D$ are in consecutive order, it follows that the vertices $i$ and $i+1$ are adjacent for all $i=0,1,2 \ldots \ldots \lambda$, as their difference is $1 \in S$, and $\operatorname{GCD}(1, n)=1$. But the vertex $i$ is not adjacent to the vertices $i+2, i+4, \ldots \ldots$ as their difference is $2,4,6 \ldots$ and $2,4,6 \ldots \notin S$, since $n$ is even, $\operatorname{GCD}(2, n)>1, G C D(4, n)>1$ and so on. Hence we cannot get a complete graph on the vertices of $D$.

Even if we form a dominating set of cardinality greater than $\lambda+1$, then also we cannot get a complete graph on this set because when $n$ is an even number, the graph $G\left(Z_{n}, \varphi\right)$ is bipartite.

Hence the clique domination number does not exist, if $n$ is an even number.

Case 2: Suppose $n$ is an odd number. Then $n=15$, as this is the first number that occurs in the prime power decomposition of $n$. Now we claim that $\lambda=2$ and $D=\{0,1,2\}$. Obviously $\operatorname{GCD}(0, n)=1, \operatorname{GCD}(1, n)=$ $1, G C D(2, n)=1$. But $\operatorname{GCD}(3, n)=G C D(3,15) \neq 1$. Hence $\lambda=2$ and $D=\{0,1,2\}$. Obviously there exists a triangle between these vertices. Therefore $D=\{0,1,2\}$ becomes a dominating clique in $G\left(Z_{n}, \varphi\right)$. This is possible only when $n=p_{1}^{\alpha_{1}} p_{2}^{\alpha_{2}}$. For other prime decompositions of $n$ the dominating set becomes an odd cycle, but not a complete graph, because the difference between the vertices is a prime which is not in $\mathrm{S}$.

Hence the dominating clique exists only when $n$ is an odd number of the form $n=p_{1}^{\alpha_{1}} p_{2}^{\alpha_{2}}$.

\section{Illustrations}

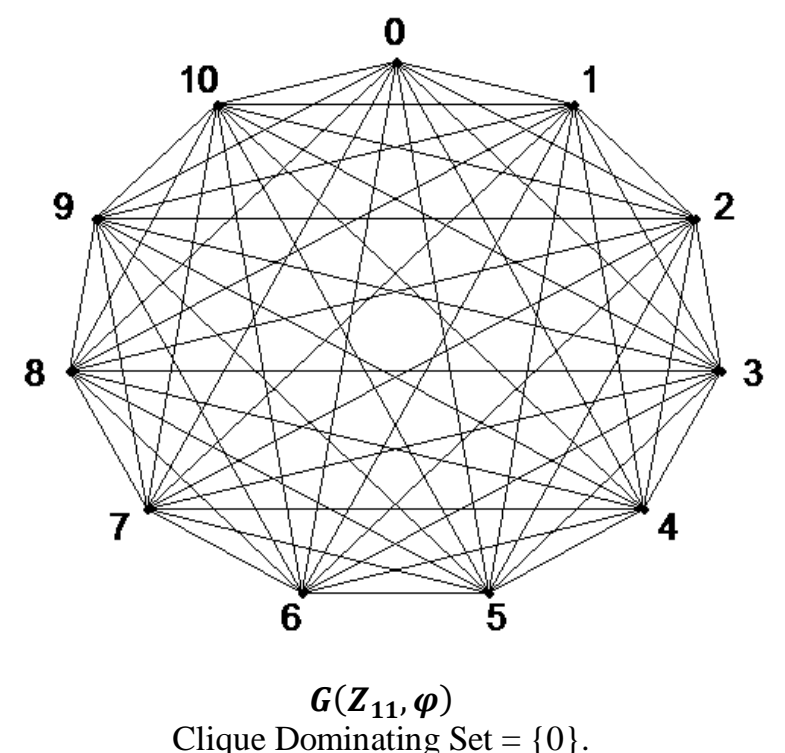

Clique Dominating Set $=\{0\}$. 


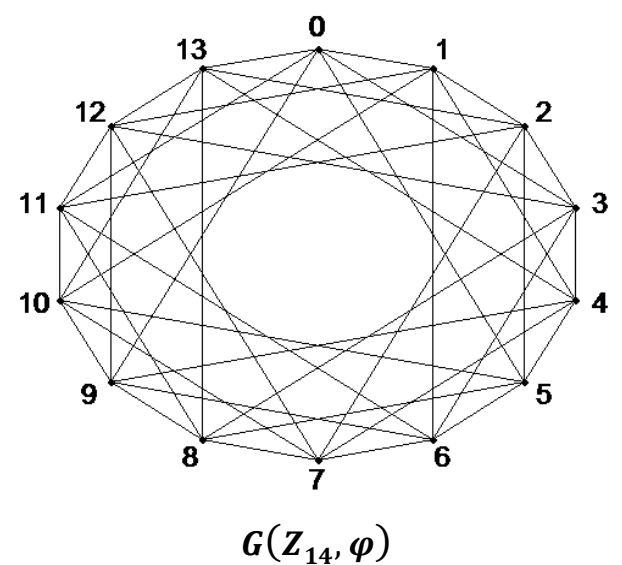

Clique Dominating Set does not exist.

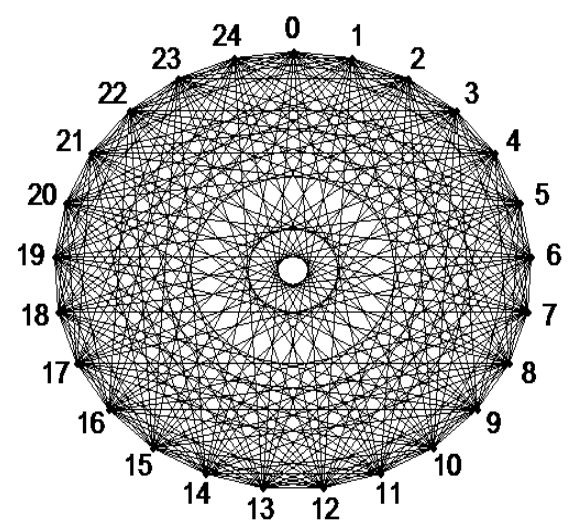

$G\left(Z_{25}, \varphi\right)$

Clique Dominating Set $=\{0,1\}$.

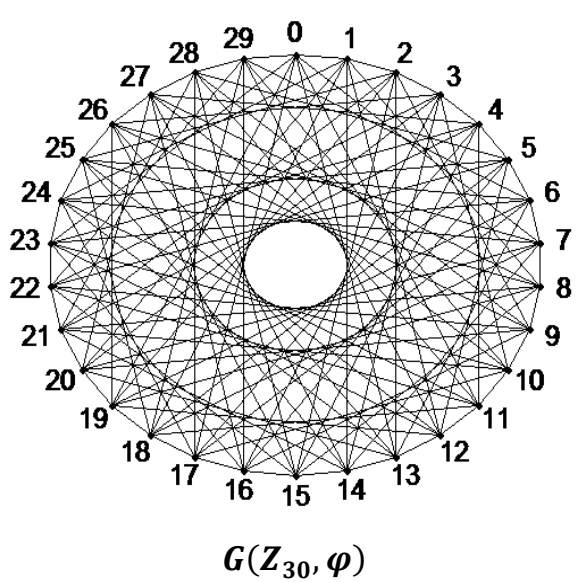

Clique Dominating Set does not exist.

\section{References}

[1]. Nathanson and B.Melvyn, Connected components of arithmetic graphs, Monat.fur.Math, 29, 1980, 219 - 220.

[2]. L.Madhavi, Studies on domination parameters and enumeration of cycles in some Arithmetic Graphs, Ph. D. Thesis submitted to S.V.University, Tirupati, India, 2002.

[3]. S.Uma Maheswari, Some Studies on the Product Graphs of Euler Totient Cayley Graphs and Arithmetic V $V_{n}$ Graphs, Ph. D. Thesis submitted to S.P.Women's University, Tirupati, India, 2012.

[4]. S.Uma Maheswari, and B.Maheswari, Domination parameters of Euler Totient Cayley Graphs, Rev.Bull.Cal.Math.Soc. 19 (2), 2011, 207-214.

[5]. O.Ore, Theory of Graphs, Amer. Math. Soc. Colloq. Publ., 38, Providence, 1962.

[6]. C. Berge, The Theory of Graphs and its Applications, Methuen, London 1962.

[7]. M.B. Cozzens, and L.L. Kelleher, Dominating cliques in graphs, Discrete Math. 86, 1990, 101-116.

[8]. G. Bacso and Z. Tuza, Dominating cliques in $\mathrm{P}_{5}$ - free graphs, Period. Math. Hungar. 21, 1990, 303-308. 